(8) Gli articoli di questa sezione sono sottoposti a referaggio doppiamente cieco (double blind peer review process) e seguono gli standard in uso per le pubblicazioni scientifiche a livello internazionale ed accettati dalle principali banche dati citazionali

\title{
Panico: nosografia di un'entità, clinica di un disturbo
}

\author{
Francesco Mancuso*, Diego Aguilar Marucco ${ }^{\circ}$,Enrico De Vivo§, Marina Bellinato", \\ Giuliano Desantis**, Alessandro Gramonis, Daniele Pini ${ }^{*}$,Daniela Zeme ${ }^{\infty}$, Emanuele Bignamini ${ }^{\circledR}$
}

\section{SUMMARY}

- Panic Disorder has not always been recognised as an exclusively psychiatric condition. Research in this area continued along medical and psychological axes until 1980, when the development of Diagnostic and Statistical Manual (DSM)-III criteria established the overall concept of panic disorder. This article briefly synthetizes the history of panic disorder until its current wording according to DSM-5 and provides elements of differential diagnosis with Panic Attacks, Agoraphobia and Substance-Related and Addictive Disorders.

The authors present a general framework for understanding the role of cognitions, emotions and physical symptoms in the pathogenesis of Panic Disorder. Recommendations for appropriate pharmacotherapy are also discussed.

Keywords: Panic Disorder, Panic Attack, Agoraphobia, Differential Diagnosis.

Parole chiave: Disturbo di Panico, Attacco di Panico, Agorafobia, Diagnosi Differenziale.

\section{Cenni storici}

Il termine "panico" affonda le sue radici nella mitologia della Grecia classica ed in particolare nella descrizione del terrore improvviso che colpiva gli uomini all'apparizione del dio Pan (Gigante e Presta, 1962).

Il quadro sintomatologico del panico compare già nelle prime descrizioni dell'isteria ad opera di Ippocrate (460-377 a.C).

Più recentemente, descrizioni di sintomi psichici e cardiorespiratori compatibili con I'attuale Disturbo di Panico (DP) possono essere rintracciate in vari testi di medicina dell'800: le "palpitazioni nervose" di Hope (1832), il "cuore irritabile di Da Costa

\footnotetext{
* Dirigente medico Ser.D. Psichiatra-Psicoterapeuta. Dottore di Ricerca in Scienze del Comportamento e dei Processi di Apprendimento. Responsabile del Centro Interdipartimentale per le Prevenzione ed il Trattamento del Tabagismo - ASL VC. E-mail: fmancuso76@gmail.com.

○ S.C.Ser.D.Torino,AslTO1.E-mail:diego.agui@libero.it.

§ S.C. 1 Ser.D.Torino, Asl TO2.E-mail: devenri@libero.it.

- S.C. 2 Ser.D. Torino, ASL TO2.E-mail: marinabellinato@ virgilio.it.

** S.C. Ser.D. Sangone, ASL TO3.E-mail: giuliano.desantis@libero.it.

c S.C.Ser.D.Pinerolo, ASLTO3.E-mail:gramoni@hotmail.com.

- S.C.Ser.D. Sangone, ASL TO3.E-mail:pinidaniele@hotmail.com. $\infty$ S.C.Ser.D. Sangone, ASL TO3.E-mail: daz72it@yahoo.it.

® S.C. 1 Ser.D. Torino, Asl TO2.E-mail: emanuele.bignamini@ aslto2.piemonte.it.
}

Fig. I - Il dio Pan

Museo del Louvre, Parigi (200 d.C.)

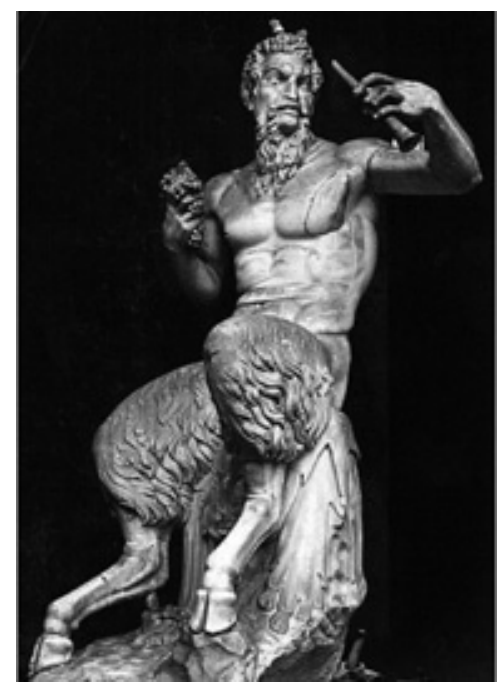

(1871), la "neuropatia cerebro-cardiaca" di Krishaber (Kaplan et al., 1996) o la "nevrosi d'angoscia" di Freud (1894).

La seconda metà del '900 si caratterizza per il progressivo affermarsi della farmacoterapia nei disturbi mentali. Klein (1964) verifica l'efficacia dell'imipramina (un antidepressivo triciclico) nel bloccare gli attacchi di panico, ma lo scarso effetto di tale molecola su altre manifestazioni ansiose. Con questa tecnica, denominata "dissezione farmacologica", Klein individua il nucleo fondamentale del DP a cui dà il nome di "ictus emotivo". 
Grazie agli studi di Klein il DP assurge ad entità clinica autonoma. Il riconoscimento ufficiale avviene nella terza edizione del Manuale Diagnostico e Statistico dei Disturbi Mentali (DSM-III) (APA, 1980) che sancisce la fine della storica concezione unitaria delle patologie ansiose delineando due distinti disturbi: il DP, rispondente a terapia con imipramina, ed il Disturbo d'Ansia Generalizzato non rispondente a tale terapia.

Sul finirie del secolo la quarta edizione del DSM (APA, 1994) è data alle stampe e, per la prima volta, viene proposto un set di criteri per definire I'Attacco di Panico (AP).

Si giunge con il nuovo millennio al DSM-5 (APA, 2013; versione italiana, 2014). L'American Psychiatric Association, oltre a decidere di abbandonare i numeri romani per identificare le edizioni, compie ulteriori e significative revisioni nosografiche, non tanto per il DP, quanto per i suoi rapporti con I'AP e I'Agorafobia.

\section{L'Attacco di Panico}

Per il DSM-IV la caratteristica essenziale di un Attacco di Panico (AP) è la comparsa improvvisa di paura o disagio intensi, che raggiunge il picco in pochi minuti, periodo durante il quale si verificano quattro o più di 13 sintomi fisici o cognitivi (set di criteri per I'AP, vedi Tabella 1 ).

\section{Tab. 1 - set di criteri per l'Attacco di Panico}

- Palpitazioni, cardiopalmo o tachicardia

- Sudorazione

- Tremori fini o a grandi scosse

- Dispnea o senso di soffocamento

- Sensazioni di asfissia

- Dolore o fastidio al petto

- Nausea o disturbi addominali

- Sensazioni di vertigine, instabilità, "testa leggera" o svenimento

- Brividi o vampate di calore

- Parestesie (sensazioni di torpore o di formicolio)

- Derealizzazione (sensazione di irrealtà, di distacco da/estraneità alla realtà circostante) o depersonalizzazione (sensazione di essere distaccati da/estranei a se stessi, alla propria mente)

- Paura di perdere il controllo o di "impazzire"

- Paura di morire

II DSM-5 mantiene sostanzialmente immutati questi criteri ma precisa: I'AP non è un disturbo mentale e può insorgere nel contesto di un DP o di un'altra patologia mentale (ad es., un differente disturbo d'ansia, un disturbo depressivo, un disturbo da uso di sostanze ecc.).

Come per l'edizione precedente, I'AP può essere anche espressione di alcune condizioni mediche generali (patologie cardiorespiratorie, ipertiroidismo, feocromocitoma, disfunzioni vestibolari, disturbi convulsivi ecc.).

Relativamente alle modalità di insorgenza, I'AP può essere descritto come: atteso ed inaspettato. Nel primo vi è un elemento scatenante al momento del verificarsi dell'attacco. Nel secondo manca tale elemento; talvolta è ancora definito attacco "a ciel sereno" (Johnson et al., 2014).

\section{Il Disturbo di Panico}

II DP può essere diagnosticato solo se in anamnesi sono presenti AP e solo se tali attacchi si caratterizzano per essere inaspettati e ricorrenti (DSM-5).
L'AP è vissuto dall'individuo come una repentina minaccia alla propria integrità psicofisica ("sto per avere un infarto", "mi sento di impazzire", "sto per perdere completamente il controllo") e può essere percepito come un segnale di qualcosa di ancor più pericoloso ("temo che i medici non abbiano capito che ho qualcosa di grave").

Tra un AP ed il successivo è tipicamente presente ansia anticipatoria, uno stato di preoccupazione continua per il possibile riproporsi di un altro AP.

Il timore per gli AP tende a modificare il comportamento quotidiano di un individuo ("dottore sono andato in vacanza lì perché c'è un ospedale vicino... sa, solo per precauzione", "dottore ho smesso di fare attività fisica perché la pressione, il cuore...").

L'ansia anticipatoria o le modifiche del comportamento devono essere presenti per almeno un mese dopo l'insorgenza di un AP. Le caratteristiche fin qui decritte danno spiegazione di alcune osservazioni sul campo: i pazienti con DP risultano essere tra i più alti utilizzatori di servizi di pronto soccorso e medicina di base (Kroenke, 2003; Marchesi et al., 2004, Bandelow et al., 2015). I primi, per la percepita minaccia alle condizioni di salute durante I'AP. I medici di famiglia, per un "controllo della pressione ogni tanto", un "controllo agli esami del sangue" o un esame strumentale che escluda una temuta condizione patologica ("dottore voglio essere sicura che non ho niente di grave").

Questa marcata preoccupazione per le condizioni di salute fisica rimanda ad una peculiarità nell'elaborazione cognitiva di questi pazienti: l'immaginazione/interpretazione catastrofica correlata a sintomi fisici anche molto blandi (Clark, 1986, 1988; APA, 2013). Una sensazione toracica è iperamplificata a segno di probabile infarto in atto. Una vertigine lascia facilmente pensare ad un tumore cerebrale. Non è una caso se gli individui affetti da DP prestino molta attenzione all'eventuale insorgenza di effetti collaterali da farmaci ("chi lo sa che cosa va a fare questo farmaco nel mio organismo", "sicuro mi fa venire qualcosa"). L'esordio del disturbo si colloca in genere tra la tarda adolescenza ed i 35 anni. La frequenza degli AP varia considerevolmente, da un attacco a settimana per mesi o più attacchi quotidiani intervallati da mesi di assenza di sintomatologia.

Se il disturbo non è trattato il decorso usualmente è cronico (anche con intervalli di remissione di anni) ed associato ad alti livelli di disabilità sociale, lavorativa e fisica.

È di frequente riscontro una diminuzione del tono dell'umore a seguito della difficoltà che si instaura nel condurre le normali attività quotidiane ("non sono più in grado di fare tutto quello che facevo un tempo", "non ho più la volontà").

Ad oggi non vi sono evidenze sufficienti per l'identificazione di un locus genetico altamente correlato alla sintomatologia del DP ed i substrati anatomofunzionali responsabili della patogenesi del disturbo rimangono ancora poco conosciuti (Perna et al., 2014; Santos et al., 2015). L'ipotesi di una disfunzione del sistema degli oppioidi endogeni o degli endocannabinoidi non ha ancora guadagnato larghi consensi (Bowers et al., 2012; Preter et al., 2014). Il fumo di sigaretta è un riconosciuto fattore di rischio per l'insorgenza del DP (Moreno et al., 2014; Bakhshaie et al., 2016). Le segnalazioni di esperienze di abuso sessuale e fisico in età infantile sono più comuni nel DP rispetto ad altri disturbi d'ansia (Pribor et al., 1992, Goodwin et al., 2005, Klauke et al., 2010).

Alcuni dati di letteratura evidenziano una maggiore prevalenza di $\mathrm{AP}$ e DP in soggetti con infezione da HBV, HCV (Carta et al., 2007) ed HIV (Bing et al., 2001; Kessler et al., 2006), rispetto a controlli sani. Sebbene il trattamento con interferone nei pazienti affetti da HCV possa esacerbare la sintomatologia del DP (Farber

continua a pagina 52 
particolare alcol, nicotina e droghe comunemente intese) e DP et al., 2005; Hosoda et al., 2000), il riscontro di una maggiore frequenza di DP nei pazienti affetti da HCV è risultato non correlato all'eventuale assunzione di terapia interferonica.

La presenza di DP è stata associata anche a più alti livelli di dolore in persone affette da HIV, lasciando ipotizzare un ruolo clinicamente significativo del DP nel determinare una minore aderenza alle terapie antiretrovirali (Tsao et al. 2004; Kosiba et al., 2014). In sintesi, I'identificazione diagnostica del DP si avvale di alcuni concetti chiave: AP ricorrenti ed inattesi (paura senza una chiara minaccia esterna), ansia anticipatoria (la paura che torni la paura) e modifiche del comportamento (vivere con i limiti imposti dalla paura).

\section{L'Agorafobia}

Il termine Agorafobia è introdotto da Westphal nel 1872 per descrivere una paura (fobia) "psicologicamente immotivata" nell'affrontare l'esposizione nella pubblica piazza (agorà) o, nell'accezione più ampia del termine, I'esposizione negli spazi aperti. Quella che appare essere una condizione fobogena opposta, la claustrofobia (paura degli spazi chiusi), in realtà presenta determinanti psicologici molto simili alla prima e cioè temere eccessivamente (o evitare) di trovarsi in situazioni in cui sia difficile (o imbarazzante) allontanarsi in caso di necessità o difficile (o imbarazzante) ricevere aiuto; indipendentemente quindi dal trovarsi in luoghi aperti o chiusi. Per tale motivo il DSM, nel corso delle varie revisioni, ha abbandonato la definizione di claustrofobia riconoscendo unicamente l'agorafobia come entità nosografica.

La paura può riguardare il rimanere fuori casa da soli, l'utilizzare trasporti pubblici, il trovarsi in spazi aperti (parcheggi, mercati, ponti ecc) o chiusi (negozi, teatri, cinema ecc.), lo stare in fila oppure tra la folla. II tipo di pensieri/paure che caratterizzano un individuo con agorafobia sono spesso: il perdersi (bambini), l'esperire sintomi di panico (adulti), una sproporzionata paura di cadere (anziani) (APA, 2013).

L'evitamento delle situazioni temute può diventare talmente grave da costringere i pazienti a non uscire più di casa, condizione che si presenta in più di un terzo dei casi. Tipicamente, il soggetto con agorafobia sceglie un accompagnatore fidato per avventurarsi fuori dalla propria abitazione. Questa figura è solitamente legata da un forte vincolo affettivo (madre, coniuge, compagno o amico).

Controversa ancora oggi è la relazione causale tra il DP e I'Agorafobia. II DSM IV inquadrava I'agorafobia con un sostanziale aggravamento del DP. II DSM-5 abbandona I'interpretazione causale e riconduce I'Agorafobia ad entità nosografia autonoma spesso ma non sempre associata al DP (Marks et al., 1987; Bienvenu et al., 2006).

Nei pazienti con DP è possibile riscontrare condotte di evitamento non gravi quanto l'Agorafobia ma comunque degne di attenzione clinica: riuscire a prendere un aereo ma preferire i posti lato corridoio ("vicino al finestrino mi sento costretto", "non so come uscirne in caso di pericolo"), non avere difficoltà ad andare al cinema o ad una conferenza ma preferire i posti vicini all'uscita di sicurezza ("mi sento più tranquilla se succede qualcosa").

\section{La diagnosi differenziale con l'uso di sostanze}

Numerose evidenze in letteratura attestano una frequente associazione tra disturbo da uso di sostanze nel corso della vita (in

(Zvolensky et al., 2006; Martins et al., 2012; Grant et al., 2015, 2016). Queste osservazioni inducono alcune riflessioni in termini di diagnosi differenziale.

L'intossicazione da stimolanti del SNC (ad es., cocaina, amfetamine, caffeina) o da cannabis e l'astinenza da depressogeni del SNC (ad es., alcol, barbiturici) possono precipitare un AP (Chou et al., 2011; Goodwin et al., 2013). Se gli AP cessano entro un arco di tempo di circa un mese dalla cessazione dell'assunzione della sostanza, è verosimile orientarsi verso una diagnosi di "disturbo d'ansia (nella fattispecie un DP) indotto da sostanze". Infatti, non viene posta diagnosi di DP se gli AP rappresentano una conseguenza fisiologica diretta dell'uso di una sostanza (es., una droga o un farmaco).

Se i sintomi di DP precedono l'esordio dell'uso di sostanze, persistono molto dopo il termine dell'astinenza acuta o dell'intossicazione grave (più di un mese) o se comunque si ha evidenza di episodi ricorrenti di AP non correlati all'uso di sostanze, è opportuno porre una diagnosi di DP in comorbilità eventuale con un disturbo da uso di sostanze.

Una diagnosi errata di "disturbo d'ansia (DP) indotto da sostanze" può essere posta nel caso di un DP che esordisce con una sintomatologia molto sfumata prima dell'uso di sostanze ma che esita in un eccessivo uso delle stesse a scopo di automedicazione. In tal caso, l'uso di sostanze può focalizzare troppo l'attenzione del clinico ed orientarlo ad un disturbo d'ansia indotto da sostanze con relativo invio al Ser.D. quando, in realtà, può trattarsi di una comorbilità tra DP e disturbo da uso di sostanze (condizione che tipicamente si riscontra in soggetti con DP e disturbo da uso di alcol lieve).

Evidenziare una comorbilità tra DP ed uso di sostanze (quindi, non unicamente un disturbo d'ansia indotto da sostanze) ha importanti risvolti in termini di coinvolgimento dei servizi e di conseguenti offerte terapeutiche (McGovern et al., 2007).

Tassi raddoppiati di insoddisfazione rispetto ad un percepito bisogno di cure sono frequentemente registrati in pazienti con comorbilità rispetto a pazienti affetti da solo disturbo da uso (Melchior et al., 2014). Spesso l'insoddisfazione è dettata proprio dalla mancanza di programmi adeguati per il trattamento simultaneo, quando in realtà, con una presa in carico congiunta, numerosi interventi integrati di provata efficacia potrebbero essere offerti (Sacks et al., 2008; 2010; 2013).

\section{Opzioni nosografiche}

Sebbene il DSM statunitense rappresenti un punto di riferimento per la formulazione della diagnosi, I'ICD 9-CM (WHO, 1979) dell'Organizzazione Mondiale della Sanità è ufficialmente utilizzato in Italia per la codifica delle informazioni cliniche rilevate dalla Scheda di Dimissione Ospedaliera (SDO) e dai flussi informativi distrettuali e domiciliari.

Per le differenze esistenti tra criteri nosografici del DP adottati dal DSM-5 ed i corrispettivi formulati dall'ICD-10 (WHO, 1992) si rinvia alla Tabella 2.

In tema di sistemi classificativi, nel 2011 la World Psychiatric Association, in collaborazione con I'Organizzazione Mondiale della Sanità, ha condotto un sondaggio selezionando in maniera randomizzata un campione di psichiatri in 44 paesi del mondo (Reed et al., 2011). L'Italia è risultata seconda sola alla Francia in termini di abitudine degli psichiatri a non utilizzare nella loro pratica clinica un sistema formale di classificazione e diagnosi dei disturbi mentali. In Francia, ha dichiarato di utilizzarlo meno del $50 \%$ degli intervistati, in Italia poco più del $60 \%$. 
A differenza del DMS-5, l'ICD-10 prevede per la diagnosi di DP:

- almeno 4 attacchi in un periodo di 4 settimane

- è esclusa diagnosi nel caso di Schizofrenia o di

- disturbo dell'umore

L'AP ha un ulteriore sintomo caratterizzante, la secchezza delle fauci, e, per poter porre diagnosi, si deve riscontrare almeno uno tra i seguenti:

- palpitazioni

- sudorazione

- tremore

- secchezza delle fauci

L'undicesima edizione dell'ICD è prevista per il 2018

In Germania oltre il 90\%. Tra gli psichiatri italiani che hanno dichiarato di utilizzare un sistema formale, il $50 \%$ ha indicato come riferimento il DSM ed il 37\% I'ICD.

Affermare di usare un sistema formale di classificazione non vuol dire automaticamente applicare regolarmente tutti i criteri relativi al numero ed alla durata dei sintomi di un disturbo (Maj, 2014). D'altronde, "i criteri diagnostici sono offerti come linee guida per porre diagnosi e il loro uso deve essere orientato dal giudizio clinico" (DSM-5, Uso del manuale).

\section{Terapia}

Tutti gli SSRI (escitalopram, citalopram, fluoxetina, fluvoxamina, paroxetina e sertralina) e gli SNRI (venlafaxina) hanno fornito convincenti prove di efficacia e sono considerati oggi farmaci di prima scelta nel trattamento del DP (Bandelow et al., 2008; Perna et al., 2011; Baldwin et al., 2014; Freire et al., 2014).

Alcuni TCA (clomipramina, desipramina, imipramina, lofepramina) hanno dimostrato pari efficacia ma i loro maggiori effetti collaterali ne limitano fortemente l'uso.

Gli inibitori selettivi del reuptake della noradrenalina (reboxetina), gli IMAO (fenelzina), gli anticonvulsivanti (gabapentin, valproato di sodio) e gli antipsicotici atipici (olanzapina, aripiprazolo, risperidone e sulpiride) possono rappresentare strategie terapeutiche alternativive sebbene non abbiano raggiunto in letteratura un unanime consenso (Batelaan et al., 2012; Freire et al., 2014, 2016; Perna et al., 2016).

Le benzodiazepine (alprazolam, clonazepam, diazepam, lorazepam) sono farmaci molto efficaci in particolare nelle manifestazioni acute. Le linee guida tendono a sconsigliare il loro utilizzo come farmaci di prima scelta (APA, 2009; NICE, 2011) per il possibile instaurarsi di dipendenza, per una possibile compromissione dell'attenzione e della memoria a breve termine o per una maggiore frequenza di eventi quali incidenti stradali e cadute (Double et al., 2000, RANZCP, 2003; Uzun, 2010).

Nonostante ciò, le benzodiazepine sono costantemente prescritte come farmaci di prima linea nel DP. Ciò è dovuto ad alcune attitudini del clinico che, talora, tende a preferire le proprie "mindlines" alle più ufficiali "guidelines". In altri termini, per la prescrizione di un farmaco si tende a dare priorità ai riscontri derivanti dalla propria pratica clinica rispetto alle raccomandazioni basate sulle evidenze (Baldwin et al., 2012).

La capacità di indurre addizione da parte delle benzodiazepine è oggi molto controversa (Lader, 2011; Baldwin et al., 2013) ed è generalmente temuta in base all'evidenza che molti individui con un'addizione abusano di benzodiazepine (Starcevic, 2014). In realtà, in assenza di disturbo da uso di altre sostanze, la dipendenza da benzodiazepine è di tipo terapeutico, una dipendenza fisica o, con un termine suggestivo, una dipendenza non additiva (Starcevic et al., 2012). Tale forma di dipendenza si connota per l'instaurarsi di tolleranza e per la comparsa di sindrome da sospensione (astinenza), all'interruzione dell'assunzione prolungata del farmaco; questi due fenomeni sono conseguenza di un normale adattamento fisiologico alla presenza di una sostanza che agisce sul SNC (Buhler et al., 2005; O'Brien et al., 2006( e si possono manifestare con un uso prolungato (sindrome da sospensione) o anche breve (tolleranza) (O'Brien et al., 2006; Baldwin et al., 2013) della stessa sostanza. A conferma di quanto sopra, la sola presenza di tolleranza o di sindrome da sospensione (astinenza) in soggetti che usano benzodiazepine regolarmente prescritte non consente di porre diagnosi di addizione da benzodiazepine in accordo ai criteri del DSM-5.

È da aggiungere che nei soggetti con uso prolungato di benzodiazepine, sempre in assenza di comorbilità presente o passata con distrubo da uso di altre sostanze, la tolleranza non si instaura costantemente (a differenza della sindrome da sospensione) (Nardi et al., 2012; Soumerai et al., 2003; Starcevic, 2014) ed è comunque molto meno marcata per gli effetti ansiolitici ed ipnoinducenti rispetto a quelli anticonvulsivanti (Balwin et al., 2013; Gravielle, 2016).

Di converso, per formulare diagnosi di addizione da benzodiazepine, devono essere presenti sintomi quali craving o uso continuativo nonostante la presenza di persistenti o ricorrenti problemi sociali o interpersonali (Starcevic 2016; Shaffer, 1999; APA, 2013). Il pattern di addizione da benzodiazepine (senza uso di altre sostanze), al pari dell'abuso (Salzman et al., 1991; Andersch et al., 2003), non è di frequente riscontro nella pratica clinica (Cook et al., 2007, Lalive et al., 2011, Starcevic, 2014).

Oltre alla farmacoterapia, un intervento di dimostrata efficacia nel DP è la psicoterapia, in particolar modo la psicoterapia cognitivocomportamentale (APA, 2009; NICE, 2011). La combinazione di antidepressivi e psicoterapia ha dato prova di maggiore efficacia rispetto agli antidepressivi od alla psicoterapia in monoterapia (Furukawa et al., 2007; Koszycki et al., 2011; Van Apeldoorn et al., 2010, Bandelow et al., 2012; Katzman et al., 2014). Notoriamente, non sempre sono presenti le condizioni per impostare una psicoterapia; un aprioristico approccio combinato non è quindi consigliato (APA, 2009). In presenza di comorbilità per moderata o severa agorafobia è opportuno invece considerare con più attenzione un approccio combinato (Van Apeldoorn et al., 2013).

La psicoterapia cognitivo-comportamentale, in una recente metanalisi, si è dimostrata maggiormente efficace rispetto ad altre terapie psicologiche quali psicoeducazione, terapia supportiva, terapie fisiologiche (tecniche di controllo del respiro, di rilassamento e di biofeedback), terapia cognitiva, mindfulness e terapie psicodinamiche. Nonostante ciò gli autori dello studio hanno sottolineato la mancanza di una prova inequivocabile a supporto della psicoterapia cognitivo-comportamentale rispetto alle altre psicoterapie (Pompoli et al., 2016).

\section{Elaborazione emozionale ed elementi di rapporto}

Oltre alle caratteristiche cognitive cui si è accennato (immaginazione catastrofica), i pazienti con DP presentano tratti tipici anche nell'elaborazione emozionale (Nemiah, 1976; Tuescher et al., 2011). Uno studio del Dipartimento di Psichiatria di Napoli ha evidenziato, in linea con alcune precedenti osservazioni (Nemiah, 
1970; Parker et al., 1993, Zeitlin et al., 1993), in un campione drug free di pazienti con DP, una maggiore frequenza di alessitimia rispetto ai controlli sani, in particolare un deficit nella capacità di percepire ed esprimere le emozioni (Galdersi et al., 2008). Tali deficit sono stati spesso associati ad una più generale difficoltà nell'elevare le emozioni da un livello sensomotorio dell'esperienza ad un livello cognitivo/rappresentativo (Nemiah, 1984a, 1984b; Lane et al., 1987). Per semplificare, bisogna considerare che un'emozione porta con sé due aspetti: un correlato fisico (ad es., la sensazione di caldo o la tachicardia nella rabbia) ed un correlato cognitivo (capire che si tratta di rabbia per i pensieri di avversione contro l'interlocutore). Un soggetto con un marcato deficit nella percezione delle emozioni si potrà arrabbiare senza capire che si tratta di rabbia ma rendendosi conto solo di un'improvvisa sensazione di calore o tachicardia (Taylor et al., 1991). In altri termini, il soggetto non riuscirà (o riuscirà poco) ad integrare vissuti emozionali nella dimensione della consapevolezza (Lane et al., 1996). Non riuscendo a mentalizzare I'esperienza emotiva (Busch et al., 2014) I'individuo potrà esperire solo I'aspetto fisico delle emozioni (Nemiah et al., 1976; Sifneos, 1988; Salovey et al., 1990; Taylor, 1992a).

Un'accelerazione improvvia e ingiustificata del battito cardiaco senza il riconoscimento di uno condizione emotiva sottostante non giustifica necessariamente una crisi di panico. Nei fatti, potrebbe solo determinare preoccupazione e programmazione di una visita medica. Nei pazienti con DP, però, è anche presente una paura delle sensazioni corporee (Clark et al., 1997) unitamente alla precedentemente citata immaginazione catastrofica. Verrebbe quindi a crearsi un corto-circuito nell'elaborazione cognitiva ed emozionale di questi pazienti: parzialmente o totalmente inconsapevoli dell'agitarsi interiore di una o più emozioni, i segnali corporei verrebbero interpretati come minacciosi. La minaccia genererebbe ansia che di per sé andrebbe ad acuire le temute sensazioni corporee (Ehlers et al., 1988a, 1992; Ehlers, 1993). Con questo circuito a feedback lo stato indifferenziato di tensione si amplificherebbe al tal punto da configurare la condizione come catastrofica (Ehlers et al., 1988b; Pauli et al., 1991; Keefe, 1995) per la minaccia totalizzante alla propria integrità fisica (Clark, 1997).

La "cecità emozionale" (Lanee et al., 1997) nella decodifica di vissuti interiori rappresenta un aspetto dell'intelligenza emozionale ed è predittrice di una maggiore gravità del disturbo (De Berardis et al., 2007). È opportuno valutare con attenzione questi aspetti (Izci et al., 2014, Zou et al., 2016) non solo per un corretto inquadramento nosografico ma anche per le importanti ripercussioni in termini psicoterapeutici. Una difficoltà nell'identificare accuratamente le emozioni si lega ad una difficoltà conseguente ad esprimerle. Una verbalizzazzione scadente dei propri vissuti emozionali determina, a sua volta, una modulazione scadente delle emozioni e, non secondariamente, una maggiore difficoltà nel riuscire a trovare nelle altre persone una fonte di aiuto per la regolazione della propria angoscia (Taylor, 1992b).

La difficoltà a parlare delle proprie emozioni è l'aspetto più manifesto degli individui alessitimici e l'etimologia stessa del termine alessitimia rimanda alla "mancanza di parole per le emozioni": a $=$ mancanza, lexis = parola, thymos = emozione (Sifneos, 1972).

Ulteriore aspetto da considerare nel rapportarsi ad un individuo affetto da DP è la percepita incomprensione. Nelle fasi iniziali della malattia le figure significative (genitori, figli, coniugi ecc) tendono in genere a garantire vicinanza. Dopo un periodo variabile di tempo, se il decorso cronicizza, si cerca l'aiuto di un medico o di uno psicologo (o qualsivoglia altra figura di riferimento per la salute culturalmente accettata). Le figure significative iniziano a non garantire più la stretta vicinanza inziando talvolta anche a sminuire il problema ("non è nulla, sei solo tu che ti impressioni", "vedi di reagire"), in un contesto in cui la richiesta di prossimità da parte del paziente risulta del tutto immutata. Oltre alla necessità di esser compreso per una sofferenza vissuta come intensa, reale e totalizzante, il paziente esprimerà forti esigenze di rassicurazione, probabilmente sin dalla prima visita, per una condizione di vita le cui prospettive appaiono altamente invalidanti (Gadermann et al., 2012). Anche il rapporto con il farmaco rifletterà aspetti di rassicurazione. Avere sempre con sé delle gocce o delle compresse di ansiolitico, senza necessariamente assumerle, assumerà valenza di talismano per affrontare la giornata con maggiore sicurezza.

Un'ulteriore messa alla prova del terapeuta verrà attuata sul piano della presa in carico. II terapeuta dovrà esser in grado di trasmettere una disposizione affidabile al farsi carico della storia che il paziente racconta attraverso la sua sofferenza ma, ancor più, dovrà trasmettere sicurezza nel garantire la propria presenza fisica nel tempo a venire poiché l'esigenza di rassicurazione può rappresentare anche un epifenomeno dell'ansia di separazione da figure di riferimento (Bowlby, 1980; Raphael, 1983) spesso presente già nell'infanzia dei soggetti con DP (Rosenbaum et al., 1993; Klein, 1995; Biederman et al., 2005; Kossowsky et al., 2013). Tale condizione si manifesta come richiesta di attenzione ed assistenza nei confronti delle figure significative e, conseguentemente, verso il terapeuta (Busch et al., 2011; Milrod et al., 2014). Eventi di separazione/perdita precedenti l'insorgenza degli AP sono di frequente riscontro in questi pazienti (Faravelli et al., 1985, Kessler et al., 1997; Peter et al., 2005; Klauke et al., 2010), a conferma di una particolare "vulnerabiltà" alla separazione (Klein, 1995; Battaglia et al., 2009, 2014). Pan, in fin dei conti, era un bambino abbandonato. La ninfa che partorì il dio mezzo uomo e mezzo caprone lo abbandonò subito dopo la nascita, colpita dalla sua deformità (Hillmann, 1977).

Quanto fin qui descritto non può non richiamare le esperienze infantili di attaccamento (Bolwby, 1989). La qualità della relazione di attaccamento e la conseguente capacità di regolazione delle emozioni da parte del bambino (Sroufe, 1983; Kobak et al., 1988), risultano essere punti nodali per lo sviluppo dell'ansia di separazione (Kagan et al., 1993; Rosenbaum et al., 1993; Milrod, 2014), dell'alessitimia (Troisi et al., 2001; Taylor, 2010) e del DP nell'adulto (Gittleman et al., 1997).

Secondo il modello cognitivo post-razionalista (Guidano, 2007) una predisposizione al DP potrebbe nascere nell'ambito di una relazione di attaccamento con genitori iperattenti, iperpremurosi e iperprotettivi. Tali genitori fornirebbero una protezione caratterizzata dallo spavento (il mondo come luogo pieno di pericoli) cosicché per il bambino sentirsi protetto (vicinanza ai genitori) comporterà il perdere la propria autonomia e libertà (esplorare il mondo). Si instaurerebbe quindi una perturbazione emotiva, uno stress, non identificabile: il bambino percepirebbe un limite all'esplorazione (il mondo è pericoloso) ma senza riuscire a percepirne le cause (poiché i genitori nei fatti appaiono interamente dedicati a lui stesso). La conseguenza per il bambino è: 1) non poter elaborare cognitivamente un fattore di stress/emozione (l'emozione non raggiunge la consapevolezza), potendolo vivere solo a livello fisico (sensazioni corporee); 2) strutturare una percezione di insicurezza nell'affrontare il mondo e 3) sentire di riuscire ad affrontare la realtà circostante solo con una figura protettiva.

Il percorso psicoterapeutico dei pazienti con DP, con una favorevole combinazione degli elementi di rapporto terapeutico, delI'attitudine dell'individuo all'elaborazione intrapsichica e del decorso clinico non eccessivamente cronicizzato, spesso produce una riduzione della sintomatologia ansiosa già nei primi incontri; condizione perlopiù transitoria. 
L'obiettivo della psicoterapia si pone sulla lunga distanza: riuscire a trasmettere un metodo per l'indagine interiore. Facilitare l'identificazione di quelle dimensione profonde che con gentilezza o irruenza periodicamente emergono alla consapevolezza per essere riconosciute. II processo è generativo poiché l'integrazione di tali dimensioni nell'immagine cosciente di sé genera un aumento della complessità del funzionamento psichico, sia in termini di aumentata percezione della realtà interiore che in termini di maggiore elaborazione nei significati che alla stessa si attribuiscono. II mutamento produrrà una parallela trasformazione in come si percepisce e nei significati che si attribuiscono alle altrui realtà psichiche. È, nei fatti, una ridefinizione complessiva del modo in cui si è in questo mondo.

Come sarà questo nuovo essere al mondo non è dato sapere. Auspicabilmente, fedele a ciò che di più intimo portiamo in noi stessi.

\section{Bibliografia}

American Psychiatric Association (APA) (1980). Diagnostic and Statistical Manual of Mental Disorders, 3rd edition (DSM-III). Washington DC: American Psychiatric Press.

American Psychiatric Association (APA) (1994). Diagnostic and Statistical Manual of Mental Disorders, 4th edition (DSM-IV). Washington DC: American Psychiatric Press

American Psychiatric Association (APA) (20139. Diagnostic and Statistical Manual of Mental Disorders, 5th edition (DSM-5). Washington DC: American Psychiatric Press.

American Psychiatric Association (APA) (2013). Manuale Diagnostico e Statistico dei Disturbi Mentali, quinta edizione (DSM-5). Milano: Raffaello Cortina Editore.

American Psychiatric Association (APA) (2009). Practice Guideline for the Treatment of Patients With Panic Disorder, 2nd edition. Washington (DC): American Psychiatric Publishing Inc.

Andersch S., Hetta J. A. (2003). 15-year follow-up study of patients with panic disorder. Eur Psychiatry, 18: 401-408.

Baldwin D.S., Talat B. (2012). Should benzodiazepines still have a role in treating patients with anxiety disorders? Hum Psychopharmacol Clin Exp., 27: 237-238.

Baldwin D.S., Aitchison K., Bateson A., Curran H.V., Davies S., Leonard B., Nutt D.J., Stephens D.N., Wilson S. (2013). Benzodiazepines: risks and benefits. A reconsideration. J. Psychopharmacol., 27(11): 967-971.

Baldwin D.S., Anderson I.M., Nutt D.J., Allgulander C., Bandelow B., den Boer J.A., Christmas D.M., Davies S., Fineberg N., Lidbetter N., Malizia A., McCrone P., Nabarro D., O'Neill C., Scott J., van der Wee N., Wittchen H.U. (2014). Evidence-based pharmacological treatment of anxiety disorders, post-traumatic stress disorder and obsessivecompulsive disorder: a revision of the 2005 guidelines from the British Association for Psychopharmacology. J Psychopharmacol, 28(5): 403439.

Bakhshaie J., Zvolensky M.J., Goodwin R.D. (2016). Cigarette smoking and the onset and persistence of panic attacks during mid-adulthood in the United States: 1994-2005. J Clin Psychiatry, 77(1): 21-24.

Bandelow B., Michaelis S. (2015). Epidemiology of anxiety disorders in the 21st century. Dialogues Clin Neurosci, 17(3): 327-335.

Bandelow B., Zohar J., Hollander E., Kasper S., Möller H.J. (2008). WFSBP Task Force on Treatment Guidelines for Anxiety, Obsessive-Compulsive and Post-Traumatic Stress Disoders, et al. World Federation of Societies of Biological Psychiatry (WFSBP) guidelines for the pharmacological treatment of anxiety, obsessive-compulsive and post-traumatic stress disorders - first revision. World J Biol Psychiatry, 9(4): 248-312.

Bandelow B., Sher L., Bunevicius R., Hollander E., Kasper S., Zohar J., Möller H.J. WFSBP Task Force on Mental Disorders in Primary Care; WFSBP Task Force on Anxiety Disorders, OCD and PTSD. (2012). Guidelines for the pharmacological treatment of anxiety disorders, obsessive-compulsive disorder and posttraumatic stress disorder in primary care. Int J Psychiatry Clin Pract, 16(2): 77-84.

Batelaan N.M., Van Balkom A.J.L.M., Stein D.J. (2012). Evidence-based pharmacotherapy of panic disorder: An update. Int J Neuropsycho pharmacol, 15: 403-415.

Battaglia M., Pesenti-Gritti P., Medland S.E., Ogliari A., Tambs K., Spatola C.A. (2009). A genetically informed study of the association between childhood separation anxiety, sensitivity to $\mathrm{CO}(2)$, panic disorder, and the effect of childhood parental loss. Arch Gen Psychiatry, 66(1): 64-71.

Battaglia M., Ogliari A., D'Amato F., Kinkead R. (2014). Early-life risk factors for panic and separation anxiety disorder: insights and outstanding questions arising from human and animal studies of $\mathrm{CO} 2$ sensitivity. Neurosci Biobehav Rev, 46(3): 455-464.

Biederman J., Petty C., Faraone S.V., Hirshfeld-Becker D.R., Henin A., Rauf A., Scott M., Pollack M., Rosenbaum J.F. (2005). Childhood antecedents to panic disorder in referred and nonreferred adults. J Child Adolesc Psychopharmacol, 15(4): 549-561.

Bienvenu O.J., Onyike C.U., Stein M.B., Chen L.S., Samuels J., Nestadt G., Eaton W.W. (2006). Agoraphobia in adults: incidence and longitudinal relationship with panic. BrJ Psychiatry, 188: 432-438.

Bing E.G., Burnam M., Longshore D. et al. (2001). Psychiatric Disorders and Drug Use Among Human Immunodeficiency Virus-Infected Adults in the United States. Arch Gen Psychiatry, 58(8): 721-728.

Bowers M.E., Choi D.C., Ressler K.J. (2012). Neuropeptide regulation of fear and anxiety: Implications of cholecystokinin, endogenous opioids, and neuropeptide. J. Physiol Behav, 107(5): 699-710.

Bowlby J. (1973). Attachment and loss. Vol. 2 Separation (1st ed), London: Hogarth Press (1980) (2nd ed). New York: Basic Books.

Bowlby J. (1989). Una base sicura. Applicazioni cliniche della teoria dell'attaccamento. Trad. Magnino M., Collana Psicologia clinica e psicoterapia n. 26. Milano: Raffaello Cortina.

Buhler K.E. (2005). Euphoria, ecstasy, inebriation, abuse, dependence, and addiction: a conceptual analysis. Med Health Care Phil, 8: 79-87.

Busch F.N., Milrod B.L., Singer M.B., Aronson A.C. (2011). Manual of Panic Focused Psychodynamic Psychotherapy: extended Range. New York: Routledge.

Busch F.N., Sandberg L.S. (2014). Unmentalized aspects of panic and anxiety disorders. Psychodyn Psychiatry, 42(2): 175-195.

Carta M.G., Hardoy M.C., Garofalo A., Pisano E., Nonnoi V., Intilla G., Serra G., Balestrieri C., Chessa L., Cauli C., Lai M.E., Farci P. (2007). Association of chronic hepatitis $\mathrm{C}$ with major depressive disorders: irrespective of interferon-alpha therapy. Clin Pract Epidemiol Ment Health, 23(3): 22

Chou K.L., Liang K., Mackenzie C.S. (2011). Binge drinking and Axis I psychiatric disorders in community-dwelling middle-aged and older adults: results from the National Epidemiologic Survey on Alcohol and Related Conditions (NESARC). J Clin Psychiatry, 72: 640-647.

Clark D.M. (1986). A cognitive approach to panic. Behav Res Ther, 24: 461 70.

Clark D.M. (1988). A cognitive model of panic attacks. In: Rachman S., Moser J.D. (Eds.). Panic: Psychological perspectives (pp. 71-89). Hillsdale, NJ: Erlbaum.

Clark D.M., Salkovskis P.M., Ost L.G., Breitholtz E., Koehler K.A., Westling B.E., Jeavons A., Gelder M. (1997). Misinterpretation of body sensations in panic disorder. J Consult Clin Psychol, 65(2): 203-213.

Cook J.M., Marshall R., Masci C., Coyne J.C. (2007). Physicians' perspectives on prescribing benzodiazepines for older adults: a qualitative study. J Gen Intern Med, 22(3): 303-307.

Curran H.V., Collins R., Fletcher S., Kee S.C.Y., Woods B., Iliffe S. (2003). Older adults and withdrawal from benzodiazepine hypnotics in general practice: effects on cognitive function, sleep, mood and quality of life. Psychol Med, 33: 1223-1227

DaCosta J.M. (1871). On irritable heart: a clinical study of a functional cardiac disorder and its consequences. Am J Medical Sciences, 61: 17-52.

De Berardis D., Campanella D., Gambi F., La Rovere R., Sepede G., Core L., Canfora G., Santilli E., Valchera A., Mancini E., Salerno R.M., Moschetta F.S., Ferro F.M. (2007). Alexithymia, fear of bodily sensations, and somatosensory amplification in young outpatients with panic disorder. Psychosomatics, 48(3): 239-246.

Doble A., Martin I. (2000). Insights into the mechanisms of benzodiazepine dependence. Eur Neuropsychopharmacol, 10: 169-169.

Ehlers A., Breuer P. (1992). Increased cardiac awareness in panic disorder. J Abnorm Psychol, 140: 164-172.

Ehlers A. (1993). Interoception and panic disorder. Advance of Behav Res Ther, 15: 3-21.

Ehlers A., Margraf J., Roth W.T., Taylor C.B., Birbaumer N. (1988a). Anxiety induced by false heart rate feedback in patients with panic disorder. Behav Res Ther, 26: 1-11.

Ehlers A., Margraf J., Roth W.T. (1988b). Selective information processing, interoception, and panic attacks. In: Hand I., Wittchen H.U. (Eds.). Panic and phobias 2 (pp. 129-148). Berlin: Springer-Verlag.

Faravelli C.,Webb T., Ambonetti A., Fonnesu F., Sessarego A. (1985). Prevalence of traumatic early life events in 31 agoraphobic patients with panic attacks. Am J Psychiatry, 142: 1493-1494. 
Farber G.A., Levin T., White C.A. (2005). A cognitive therapy conceptualization of panic disorder exacerbated by interferon treatment. Gen Hosp Psychiatry, Sep-Oct, 27(5): 329-37.

Freire R.C., Machado S., Arias-Carrión O., Nardi A.E. (2014). Current pharmacological interventions in panic disorder. CNS Neurol Disord Drug Targets, 13(6): 1057-1065.

Freire R.C., Zugliani M.M., Garcia R.F., Nardi A.E. (2016). Treatment-resistant panic disorder: a systematic review. Expert Opin Pharmacother, 17(2): 159-168.

Freud S. (1950). The justification for detaching from neurasthenia a particular syndrome: the anxiety neurosis (1894). Collected papers, vol. 1. London: Hogart Press, pp. 76-127.

Furukawa T.A., Watanabe N., Churchill R. (2007). Combined psychotherapy plus antidepressants for panic disorder with or without agoraphobia. Cochrane Database Syst Rev, 24(1): CD004364.

Gadermann A.M., Alonso J., Vilagut G., Zaslavsky A.M., Kessler R.C. (2012). Comorbidity and disease burden in the National Comorbidity Survey Replication (NCS-R). Depress Anxiety, 29: 797-806.

Galderisi S., Mancuso F., Mucci A., Garramone S., Zamboli R., Maj M. (2008). Alexithymia and cognitive dysfunctions in patients with panic disorder. Psychother Psychosom, 77(3): 182-188.

Gigante M., Presta A. (1962). PAN. Antologia della letteratura greca. Messina: D'Anna.

Gittelman Klein R. (1997). Separation Anxiety and Adult Anxiety Disorders. Giorn Ital Psicopat, 1: 4-10.

Goodwin R.D., Fergusson D.M., Horwood L.J. (2005). Childhood abuse and familial violence and the risk of panic attacks and panic disorder in young adulthood. Psychol Med, 35: 881-890.

Goodwin R.D., Stein D.J. (2013). Anxiety disorders and drug dependence: evidence on sequence and specificity among adults. Psychiatry Clin Neurosci., 67(3): 167-173.

Grant B.F., Saha T.D., Ruan W.J., Goldstein R.B., Chou S.P., Jung J., Zhang H., Smith S.M., Pickering R.P., Huang B., Hasin D.S. (2016). Epidemiology of DSM-5 Drug Use Disorder: Results From the National Epidemiologic Survey on Alcohol and Related Conditions-III. JAMA Psychiatry, 73(1): 39-47.

Grant B.F., Goldstein R.B., Saha T.D., Chou S.P., Jung J., Zhang H., Pickering R.P., Ruan W.J., Smith S.M., Huang B., Hasin D.S. (2015). Epidemiology of DSM-5 Alcohol Use Disorder: Results From the National Epidemiologic Survey on Alcohol and Related Conditions III. JAMA Psychiatry, 72(8): 757-66.

Gravielle M.C. (2016). Activation-induced regulation of GABAA receptors: Is there a link with the molecular basis of benzodiazepine tolerance? Pharmacol Res, 109: 92-100.

Guidano V. (2007). Psicoterapia cognitiva post-razionalista. Una ricognizione della teoria alla clinica. Milano: FrancoAngeli.

Hillman J. (1977). Saggio su Pan. Milano: Adelphi.

Hope J.A. (1832). A Treatise on the Disease of the Heart and Great Vessels Comprising a New View of the Physiology of the Heart's Action. According to Which the Physical Signs are Explained. London: Churchill.

Hosoda S., Takimura H., Shibayama M., Kanamura H., Ikeda K., Kumada H. (2000). Psychiatric symptoms related to interferon therapy for chronic hepatitis C: clinical features and prognosis. Psychiatry Clin Neurosci, 54(5): 565-72.

Izci F., Gültekin B.K., Saglam S., Koc M.I., Zincir S.B., Atmaca M. (2014). Temperament, character traits, and alexithymia in patients with panic disorder. Neuropsychiatr Dis Treat, 16(10): 879-85.

Johnson P.L., Federici L.M., Shekhar A. (2014). Etiology, triggers and neurochemical circuits associated with unexpected, expected, and laboratory-induced panic attacks. Neurosci Biobehav Rev, 46(3): 429454.

Kagan J., Reznick J.S., Snidman N. (1993). Biological bases of childhood: a risk factor for anxiety disorders. Harv Rev Psychiatry, 1: 2-16.

Kaplan H.I., Sadock B.J., Grebb J.A. (1996). Psichiatria: manuale di scienze del comportamento e psichiatria clinica. Torino: Centro Scientifico Internazionale.

Katzman M.A., Bleau P., Blier P., Chokka P., Kjernisted K., Van Ameringen M., Canadian Anxiety Guidelines Initiative Group on behalf of the Anxiety Disorders Association of Canada/Association Canadienne des troubles anxieux and McGill University, Antony M.M., Bouchard S., Brunet A., Flament M., Grigoriadis S., Mendlowitz S., O'Connor K., Rabheru K., Richter P.M., Robichaud M., Walker J.R. (2014). Canadian clinical practice guidelines for the management of anxiety, posttraumatic stress and obsessive-compulsive disorders. BMC Psychiatry, 14(Suppl 1): S1.

Keefe R.S.E. (1995). The contribution of neuropsychology to psychiatry. Am J Psychiatry, 152: 6-15.
Kessler R.C., Davis C.G., Kendler K.S. (1997). Childhood adversity and adult psychiatric disorder in the US National Comorbidity Survey. Psychol Med, 27: 1101-1119.

Kessler R.C., Berglund P., Demler O., Jin R., Merikangas K.R., Walters E.E. (2005). Lifetime prevalence and ageof-onset distributions of DSM-IV disorders in the National Comorbidity Survey Replication. Arch Gen Psychiatry, 62: 593-602.

Kessler R.C., Chiu W.T., Jin R., Ruscio A.M., Shear K., Walters E.E. (2006). The epidemiology of panic attacks, panic disorder, and agoraphobia in the National Comorbidity Survey Replication. Arch Gen Psychiatry, 63(4): 415-424.

Klauke B., Deckert J., Reif A., Pauli P., Domschke K. (2010). Life events in panic disorder-an update on "candidate stressors". Depress Anxiety, 27(8): 716-730

Klein D.F. (1964). Delineation of two drug-responsive syndromes. Psychopharmacologia, 53: 397-408.

Klein R.G. (1995). Is panic disorder associated with childhood separation anxiety disorder? Clin Neuropharmacol, 18(Suppl 2): 7-14.

Kobak R.R., Sceery A. (1988). Attachment in late adolescence: working models, affect regulation, and representation of self and others. Child Dev, 59: 135-46.

Kosiba J.D., Gonzalez A., O'Cleirigh C., Safren S.A. (2014). Medication Adherence and HIV Symptom Distress in Relation to Panic Disorder Among HIV-Positive Adults Managing Opioid Dependence. Cognit Ther Res, 38(4): 458-464.

Kossowsky J., Pfaltz M.C., Schneider S., Taeymans J., Locher C., Gaab J. (2013). The separation anxiety hypothesis of panic disorder revisited: a meta-analysis. Am J Psychiatry, 170(7): 768-781.

Koszycki D., Taljaard M., Segal Z., Bradwejn J. (2011). A randomized trial of sertraline, self-administered cognitive behavior therapy, and their combination for panic disorder. Psychol Med, 41(2): 373-383.

Krystal H., Raskin H. (1970). Drug dependence: aspects of ego function. Detroit, MI: Wayne State University Press.

Kolb B., Whishaw I.Q. (1990). Fundamentals of human neuropsychology, 3rd ed. New York: Freeman.

Kroenke K. (2003). Patients presenting with somatic complaints: epidemiology, psychiatric comorbidity and management. Int J Methods Psychiatr Res, 12: 34-43.

Lader M. (2011). Benzodiazepines revisited-will we ever learn? Addiction, 106(12): 2086-2109.

Lalive A.L., Rudolph U., Lüscher C., Tan K.R. (2011). Is there a way to curb benzodiazepine addiction? Swiss Med Wkly, (19): 141, w13277.

Lane R.D., Schwartz G.E. (1987). Levels of emotional awareness: a cognitive developmental theory and its application to psychopathology. Am J Psychiatry, 144: 133-143.

Lane R.D., Sechrest L., Reidel R.G., Weldon V., Kaszniak A.W., Schwartz G.E. (1996). Impaired verbal and non verbal emotion recognition in alexithymia. Psychosom Med, 58: 203-210.

Lane R.D., Ahern G.L., Schwartz G.E., Kaszniak A.W. (1997). Is alexithymia the emotional equivalent of blindsight? Biol Psychiatry, 42: 834-844.

Maj M. (2014). Introduzione all'edizione italiana. In: Manuale diagnostico e statistico dei disturbi mentali - Quinta edizione (DSM-5). American Psychiatric Association. Milano: Raffaello Cortina.

Marchesi C., Brusamonti E., Borghi C., Giannini A., Di Ruvo R., Minneo F., Quarantelli C., Maggini C. (2004). Anxiety and depressive disorders in an emergency department ward of a general hospital: a control study. Emerg Med J, 21: 175-179.

Marks I.M. (1987). The agoraphobic syndrome (panic disorder with agoraphobia). In: Fears, Phobias, and Rituals. Panic, Anxiety, and Their Disorders (pp. 323-361). NewYork: Oxford University Press.

Martins S.S., Fenton M.C., Keyes K.M., Blanco C., Zhu H., Storr C.L. (2012). Mood and anxiety disorders and their association with nonmedical prescription opioid use and prescription opioid-use disorder: longitudinal evidence from the National Epidemiologic Study on Alcohol and Related Conditions. Psychol Med, 42(6): 1261-1272.

McGovern M.P., Clark R.E., Samnaliev M. (2007). Co-occurring psychiatric and substance use disorders: a multistate feasibility study of the quadrant model. Psychiatr Serv, 58: 949-954.

Melchior M., Prokofyeva E., Younès N., Surkan P.J., Martins S.S. (2014). Treatment for illegal drug use disorders: the role of comorbid mood and anxiety disorders. BMC Psychiatry, 26(14): 89

Milrod B., Markowitz J.C., Gerber A.J., Cyranowski J., Altemus M., Shapiro T., Hofer M., Glatt C. (2014). Childhood separation anxiety and the pathogenesis and treatment of adult anxiety. Am J Psychiatry, 171(1): 34-43.

Moreno-Peral P., Conejo-Cerón S., Motrico E., Rodríguez-Morejón A., Fernández A., García-Campayo J., Roca M., Serrano-Blanco A., RubioValera M., Bellón J.Á. (2014). Risk factors for the onset of panic and 
generalised anxiety disorders in the general adult population: a systematic review of cohort studies. J Affect Disord, 168: 337-348.

National Collaborating Centre for Mental Health, National Collaborating Centre for Primary Care (2011). Generalised anxiety disorder and panic disorder (with or without agoraphobia) in adults: management in primary, secondary and community care. London: National Institute for Health and Clinical Excellence (NICE).

Nardi A.E., Freire R.C., Mochcovitch M.D., Amrein R., Levitan M.N., King A.L., Valença A.M., Veras A.B., Paes F., Sardinha A., Nascimento I., deMelo-Neto V.L., Dias G.P., E Silva A.C., Soares-Filho G.L., da Costa R.T., Mezzasalma M.A., de Carvalho M.R., de Cerqueira A.C., Hallak J.E., Crippa J.A., Versiani M. (2012). A randomized, naturalistic, parallel-group study for the long-term treatment of panic disorder with clonazepam or paroxetine. J Clin Psychopharmacol, 32: 120-126.

Nemiah J.C., Freyberger H., Sifneos P.E. (1976). Alexithymia: A view of the psychosomatic process. In: Hill O.W. Modern Trends in Psychosomatic Medicine, vol. 3 (pp. 430-439). London: Butterworths.

Nemiah J.C., Sifneos P.E. (1970). Affect and fantasy in patients with psychosomatic disorders. In: Hill O.W. (Ed.). Modern Trends in Psychosomatic Medicine, vol. 2 (pp. 26-34). London: Butterworths.

Nemiah J.C. (1984a). Anxiety and psychodynamic theory. In: Grinspoon L. (Ed.). Psychiatry Update: The American Psychiatric Annual Review, vol. 3 (pp. 426-440). Washington, DC: American Psychiatric Press.

Nemiah J.C. (1984b). The psychodynamic view of anxiety. In: Pasnau R.O. (Ed.). Diagnosis and treatment of anxiety disorder. Washington, DC: American Psychiatric Press.

O'Brien C.P., Volkow N., Li T.-K. (2006). What's in a word? Addiction versus dependence in DSMV. Am J Psychiatry, 163: 764-765.

Parker J.D.A., Taylor G.J., Bagby R.M., Acklin M.W. (1993). Alexithymia in panic disorder and simple phobia: a comparative study. Am J Psychiatry, 150: 1105-1107.

Pauli P., Marquardt C., Hartl L., Nutzinger D.O., Holzl R., Strian F. (1991). Anxiety induced by cardiac perceptions in patients with panic attacks: a field study. Behav Res Ther, 29: 137-45

Perna G., Guerriero G., Brambilla P., Caldirola D. (2014). Panic and the brainstem: clues from neuroimaging studies. CNS Neurol Disord Drug Targets, 13(6): 1049-56.

Perna G., Guerriero G., Caldirola D. (2011). Emerging drugs for panic disorder. Expert Opin. Emerg Drugs, 16: 631-645.

Perna G., Alciati A., Balletta R., Mingotto E., Diaferia G., Cavedini P., Nobile M., Caldirola D. (2016). Is There Room for Second-Generation Antipsychotics in the Pharmacotherapy of Panic Disorder? A Systematic Review Based on PRISMA Guidelines. Int J Mol Sci, 13: 17(4).

Peter H., Bruckner E., Hand I., Rufer M. Childhood (2005). Separation anxiety and separation events in women with agoraphobia with or without panic disorder. Can J Psychiatry, 50: 941-944.

Pompoli A., Furukawa T.A., Imai H., Tajika A., Efthimiou O., Salanti G. (2016). Psychological therapies for panic disorder with or without agoraphobia in adults:a network meta-analysis. Cochrane Database Syst Rev, 13(4): CD011004

Preter M., Klein D.F. (2014). Lifelong opioidergic vulnerability through early life separation: a recent extension of the false suffocation alarm theory of panic disorder. Neurosci Biobehav Rev, 46(3): 345-351.

Pribor E.F., Dinwiddie S.H. (1992). Psychiatric correlates of incest in childhood. Am J Psychiatry, 149: 52-56.

Raphael B. (1983). The anatomy of bereavement. New York: Basic Books.

Reed G.M., Mendonça Correia J., Esparza P., Saxena S., Maj M. (2011). The WPA-WHO Global Survey of Psychiatrists' Attitudes Towards Mental Disorders Classification. World Psychiatry, 10(2):118-131.

Rosenbaum J.F., Biederman J., Bolduc-Murphy E.A., Faraone S.V., Chaloff J., Hirshfeld D.R., Kagan J. (1993). Behavioral inhibition in childhood: a risk factor for anxiety disorders. Harv Rev Psychiatry, 1: 2-16.

Royal Australia and New Zealand College of Psychiatrists (2003). Australian and New Zealand clinical practice guidelines for the treatment of panic disorder and agoraphobia. Aust N Z J Psychiatry, 37: 641-656.

Sacks S., Chaple M., Sirikantraporn J., Sacks J.Y., Knickman J., Martinez J. (2013). Improving the capability to provide integrated mental health and substance abuse services in a state system of outpatient care. J Subst Abuse Treat, 44: 488-493.

Sacks S., McKendrick K., Sacks J.Y., Cleland C.M. (2010). Modified therapeutic community for co-occurring disorders: single investigator meta analysis. Subst Abus, 31: 146-161.

Sacks S., McKendrick K., Sacks J.Y., Banks S., Harle M. (2008). Enhanced outpatient treatment for co-occurring disorders: main outcomes. J Subst Abuse Treat, 34: 48-60.

Salzman C. (1991). The APA Task Force report on benzodiazepine dependence, toxicity, and abuse. Am J Psychiatry, 148: 151-2.
Salovey P., Mayer J.D. (1990). Emotional intelligence. Imagination, Cognition and Personality, 9: 185-211.

Santos M., D'Amico D., Dierssen M. (2015). From neural to genetic substrates of panic disorder: Insights from human and mouse studies. Eur J Pharmacol, 759: 127-141.

Shaffer H.J. (1999). Strange bedfellows: a critical view of pathological gambling and addiction. Addiction, 94(10): 1445-1448.

Sifneos P.E. (1972). Short-Term Psychotherapy and Emotional Crisis. Cambridge. MA: Harvard University Press.

Sifneos P.E. (1988). Alexithymia and its relationship to hemispheric specialization, affect, and creativity. Psychiatric Clinics of North America, 11: 287-292.

Soumerai S.B., Simoni-Wastila L., Singer C., et al. (2003). Lack of relationship between long-term use of benzodiazepines and escalation to high dosages. Psychiatr Services, 54: 1006-1011.

Sroufe L.A. (1983). Infant-caregiver attachment in the pre-school: the roots of competence and maladaptation. In: Perlmutter M. (Ed.). Development and policy concerning children with special needs. Hove: Lawrence Erlbaum Associated.

Starcevic V. (2016). The importance of distinguishing between dependence and addiction in the context of long-term benzodiazepine use. Aust $N Z$ J Psychiatry, Apr 18, pii: 0004867416644980.

Starcevic V. (2014). The reappraisal of benzodiazepines in the treatment of anxiety and related disorders. Expert Rev Neurother., 14(11): 1275-1286.

Starcevic V. (2012). Benzodiazepines for anxiety disorders: maximising the benefits and minimising the risks. Advances in psychiatric treatment, 18: 250-258.

Taylor G.J., Bagby R.M., Parker J.D.A. (1991). The alexithymia construct; apotential paradigm for psychosomatic medicine. Psychosomatics, 32: 153-164.

Taylor G.J. (1992a). Psychoanalysis and psychosomatics: a new synthesis. J Am Acad Psychoanal, 20: 251-275.

Taylor G.J. (1992b). The alexithymia construct: conceptualization, validation, and relationship with basic dimensions of personality. International Workshop on Alexithymia, 4th National Congress of the Italian Society of Medical Psychology. Pavia.

Taylor G.J. (2010). Affects, trauma, and mechanisms of symptom formation: a tribute to John C. Nemiah, MD (1918-2009). Psychother Psychosom, 79(6): 339-349.

Troisi A., D'argenio A., Peracchio F., Petti P. (2001). Insicure attachment and alexithymia in young men with mood symptoms. J Nerv Ment Dis, 189: 311-316.

Tsao J.C., Dobalian A., Naliboff B.D. (2004). Panic disorder and pain in a national sample of persons living with HIV. Pain, 109: 172-180.

Tuescher O., Protopopescu X., Pan H. et al. (2011). Differential activity of subgenual cingulate and brainstem in panic disorder and PTSD. J Anxiety Disord, 25(2): 251-257

Uzun S., Kozumplik O., Jakovljeviç M., Sediç B. (2010). Side effects of treatment with benzodiazepines. Psychiatr Danub, 22(1): 90-93.

Van Apeldoorn F.J., Van Hout W.J., Timmerman M.E., Mersch P.P., den Boer J.A. (2013). Rate of improvement during and across three treatments for panic disorder with or without agoraphobia: cognitive behavioral therapy, selective serotonin reuptake inhibitor or both combined. J Affect Disord., 150(2): 313-319.

Van Apeldoorn F.J., Timmerman M.E., Mersch P.P.A., van Hout W.J., Visser S., van Dyck R., den Boer J.A. (2010). A randomized trial of cognitivebehavioral therapy or selective serotonin reuptake inhibitor or both combined for panic disorder with or without agoraphobia: Treatment results through 1-year follow-up. J Clin Psychiatry, 71: 574-586.

Westphal C. (1872). Die Agoraphobie: eine neuropathische Erscheinung. Arkhivder Psychiatrie, 3: 138-161.

World Health Organization (1979). Manual of the International Classification of Diseases, Injuries, and Causes of Death, 9th revision Clinical Modification. Commission on Professional and Hospital Activities. Mich.: Ann Arbor. Versione italiana: www.salute.gov.it/ imgs/C_17_pubblicazioni_2251_allegato.pdf.

World Health Organization (1992). International Classification of Diseases: Clinical Descriptions and Diagnostic Guidelines, 10th revision. Geneva: WHO.

Zeitlin S.B., McNally R.J. (1993). Alexithymia and anxiety sensitivity in panic disorder and obsessive-compulsive disorder. Am J Psychiatry, 150(4): 658-60.

Zou Z., Huang Y., Wang J., He Y., Min W., Chen X., Wang J., Zhou B. (2016). Association of childhood trauma and panic symptom severity in panic disorder: Exploring the mediating role of alexithymia. J Affect Disord, 21(206): 133-139.

Zvolensky M.J., Bernstein A., Marshall E.C., Feldner M.T. (2006). Panic attacks, panic disorder, and agoraphobia: associations with substance use, abuse, and dependence. Curr Psychiatry Rep, 8: 279-285. 